\title{
Triangulating Nucleic Acid Conformations Using Multicolor Surface Energy Transfer
}

\section{Supporting Information}

Ryan A. Riskowski ${ }^{1}$, Rachel E. Armstrong ${ }^{2}$, Nancy L. Greenbaum ${ }^{3}$, Geoffrey F. Strouse ${ }^{1,2 *}$

${ }^{1}$ Molecular Biophysics Program, Florida State University, Tallahassee, FL 32306, United States

${ }^{2}$ Department of Chemistry and Biochemistry, Florida State University, Tallahassee, FL 32306. United States

3Department of Chemistry and Biochemistry, Hunter College and The Graduate Center of the City University of New York, New York, NY 10065, United States

\section{Supporting Information}

I. McSET Complex Assembly
A. Scenario I (Supporting Figure SF1)
B. KCN Etch (Supporting Figure SF2, SF3)
C. Scenario II (Supporting Figure SF4)

II. Scattering Contribution to Lifetime Data (Supporting Figure SF5)

III. FRET-Only and SET-Only data
A. Absorbance and Emission Spectra (Supporting Figure SF6)
B. Scheme I Fluorescence Decay (Supporting Figure SF7)
C. Scheme II Fluorescence Decay (Supporting Figure SF8)
D. Hammerhead ribozyme FRET-Only and SET-Only Results (Supporting Table ST1, ST2)

IV. Energy Transfer Calculations
A. Derivation of McSET Formula
B. Calculation of $d_{0}$ and $R_{0}$
C. Constants for SET and FRET Calculations (Supporting Table ST3, ST4) 


\section{Characterization of Nucleic Acid Complex Assembly}

\section{A. Linear DNA Assembly (Scenario I)}

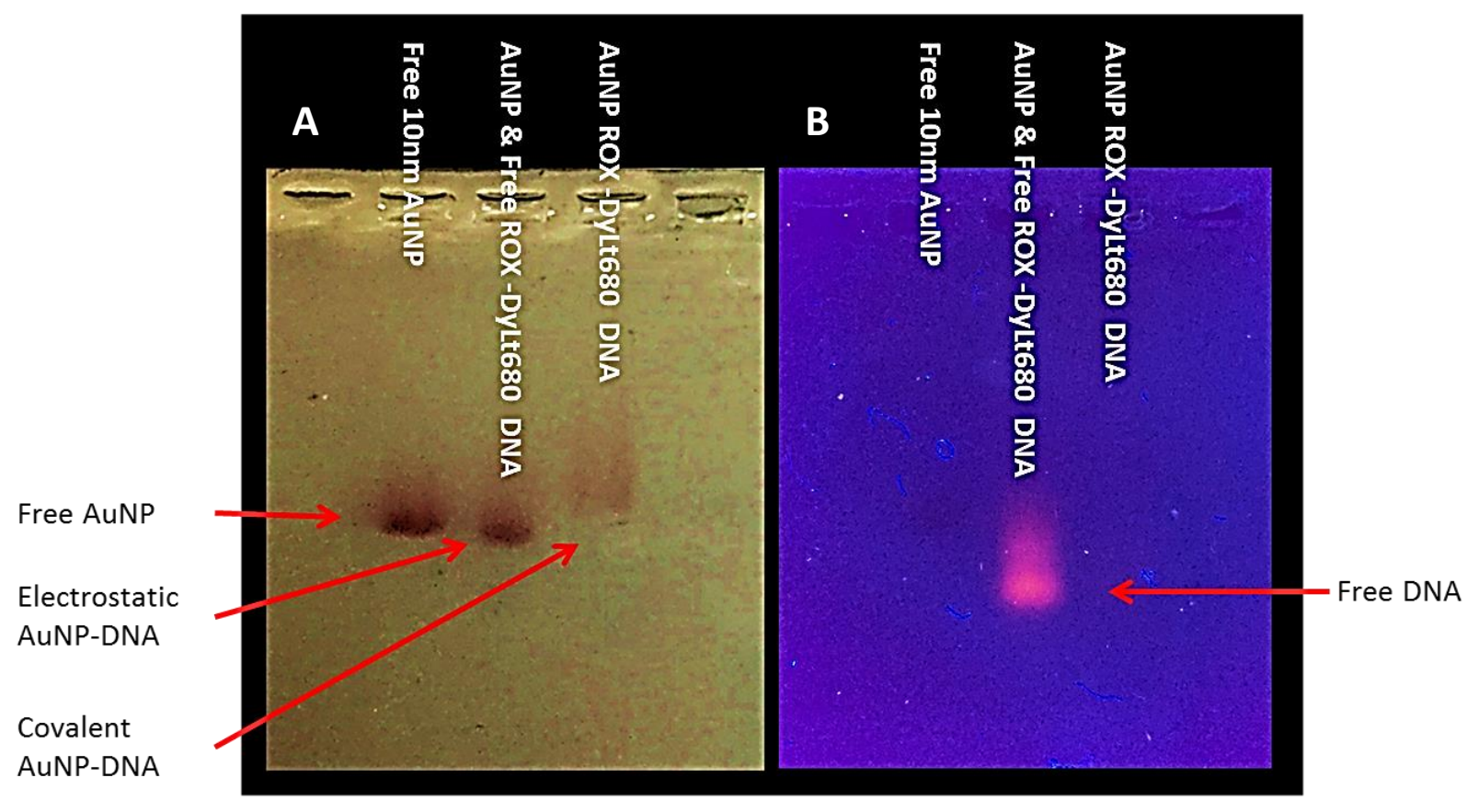

Figure SF1: $1 \%$ Agarose Gel in 1x TBE running buffer at $10 \mathrm{~V} / \mathrm{cm}$, showing assembly of the linear DNA construct used in Scenario I. Lane numbering from the left is the same in both images; Lane 1: unlabeled BSPP-coated $10 \mathrm{~nm}$ Au nanoparticles. Lane 2: unlabeled BSPP-coated $10 \mathrm{~nm}$ AuNP mixed with unbound ROX and DyLt680-labeled dsDNA. Lane 3: Fully assembled $10 \mathrm{~nm}$ AuNP covalently bound to ROX- and DyLt680-labeled dsDNA. A) Reflected light image showing bands of gold nanoparticles. The reduced rate of mobility in the gel indicates gold nanoparticles covalently bound to DNA. Smearing of the band is due to a distribution in label occupancy on the particle surface ${ }^{a}$. B) UV illuminated gel image showing dye-labeled DNA. The presence of a clear DNA band is indication of unattached DNA. The lack of a clearly visible DNA band in the covalently attached sample is the result of quenching by the AuNP and indicates very low levels of unbound DNA. Image contrast enhanced in Microsoft PowerPoint.

\footnotetext{
a Pellegrino, T.; Sperling, R. A.; Alivisatos, A. P.; Parak, W. J. Gel Electrophoresis of Gold-DNA Nanoconjugates. J Biomed Biotechnol (2007), 2007, 26796.
} 


\section{B. Determining DNA labeling efficiency by $\mathrm{CN}$ etching.}

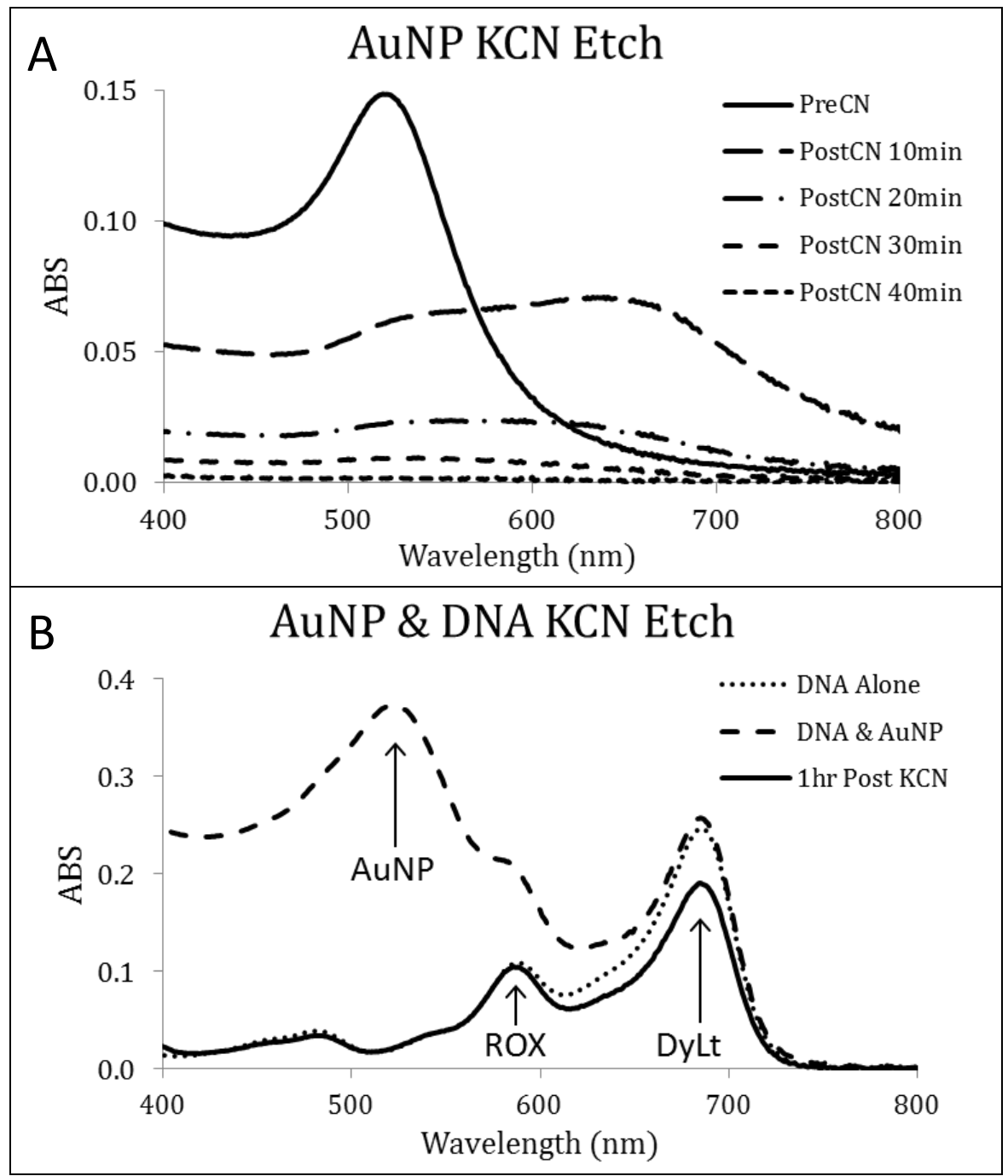

Figure SF2: Absorbance data from KCN etch control studies of A) $10 \mathrm{~nm}$ AuNP only, and B) 10 $\mathrm{nm}$ AuNP attached to dye labeled dsDNA. AuNP exhibits a transient absorbance that disappears within 40 minutes after addition of $80 \mathrm{mM} \mathrm{KCN}$. Labeling of individual traces is explained in each image. To avoid convolution with this transient absorbance, quantification of DNA was made $1 \mathrm{hr}$ after KCN addition. AuNP etching in the presence of dye labeled DNA leads to a consistent $23 \%$ decrease in absorbance for DyLt680, but ROX remains unchanged. Absorbance values were corrected for concentration. 


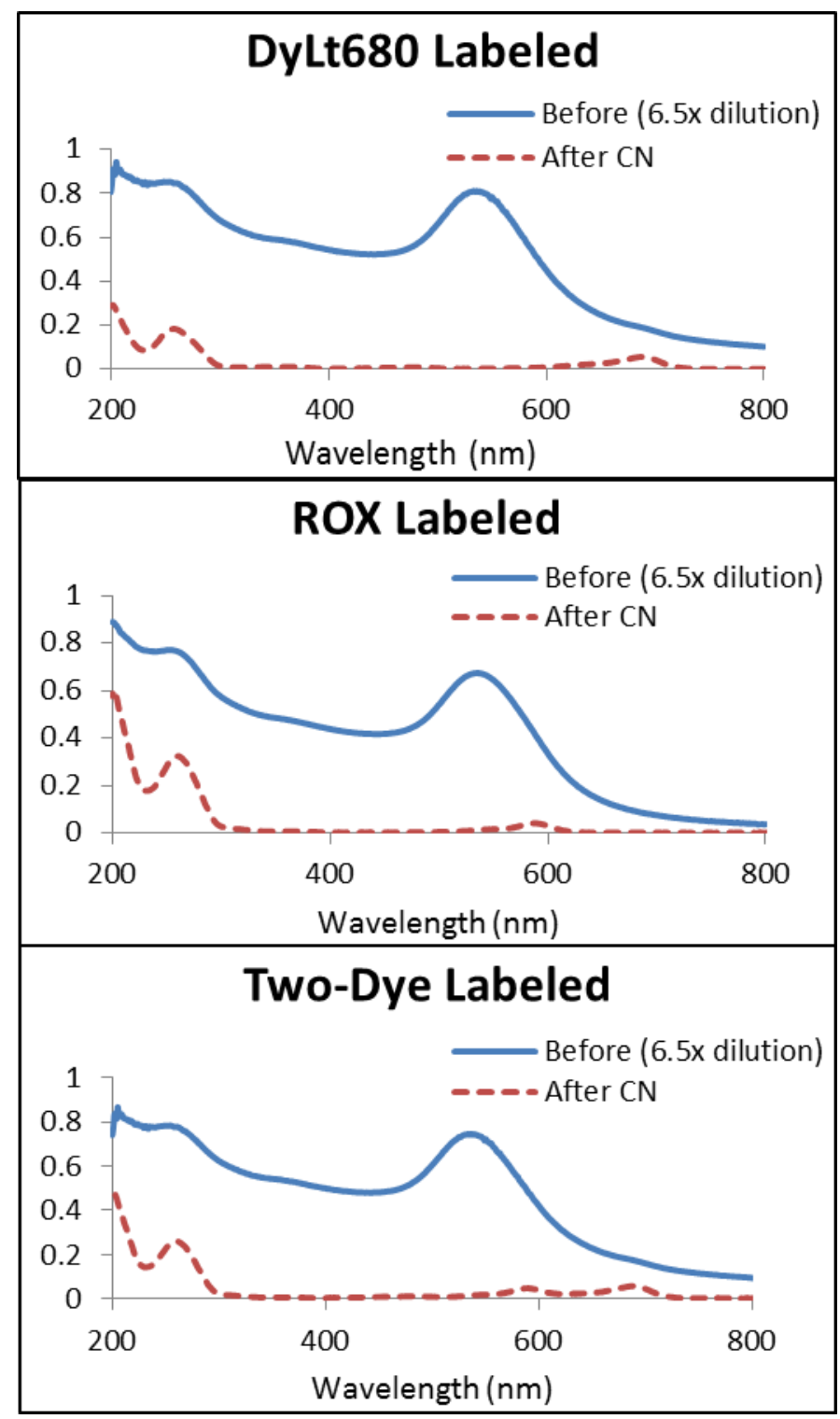

Figure SF3: Absorbance data used to determine stoichiometry of DNA duplex per AuNP in Scenario I. Strands per particle are determined from the ratio of DNA/NP concentration as determined by extinction. Nanoparticle concentration is determined from the plasmon peak at $520 \mathrm{~nm}$ before $\mathrm{CN}$ etching, and DNA concentration is determined from the dye label extinctions taken after $\mathrm{CN}$ etching. When using DyLt680 post-KCN absorbance to quantify labeling efficiency, corrections are made to account for the $23 \%$ decrease in absorbance. Average labeling efficiency was $\sim 9$ DNA duplexes per particle. 


\section{RNA Hammerhead Ribozyme Assembly (Scenario II)}

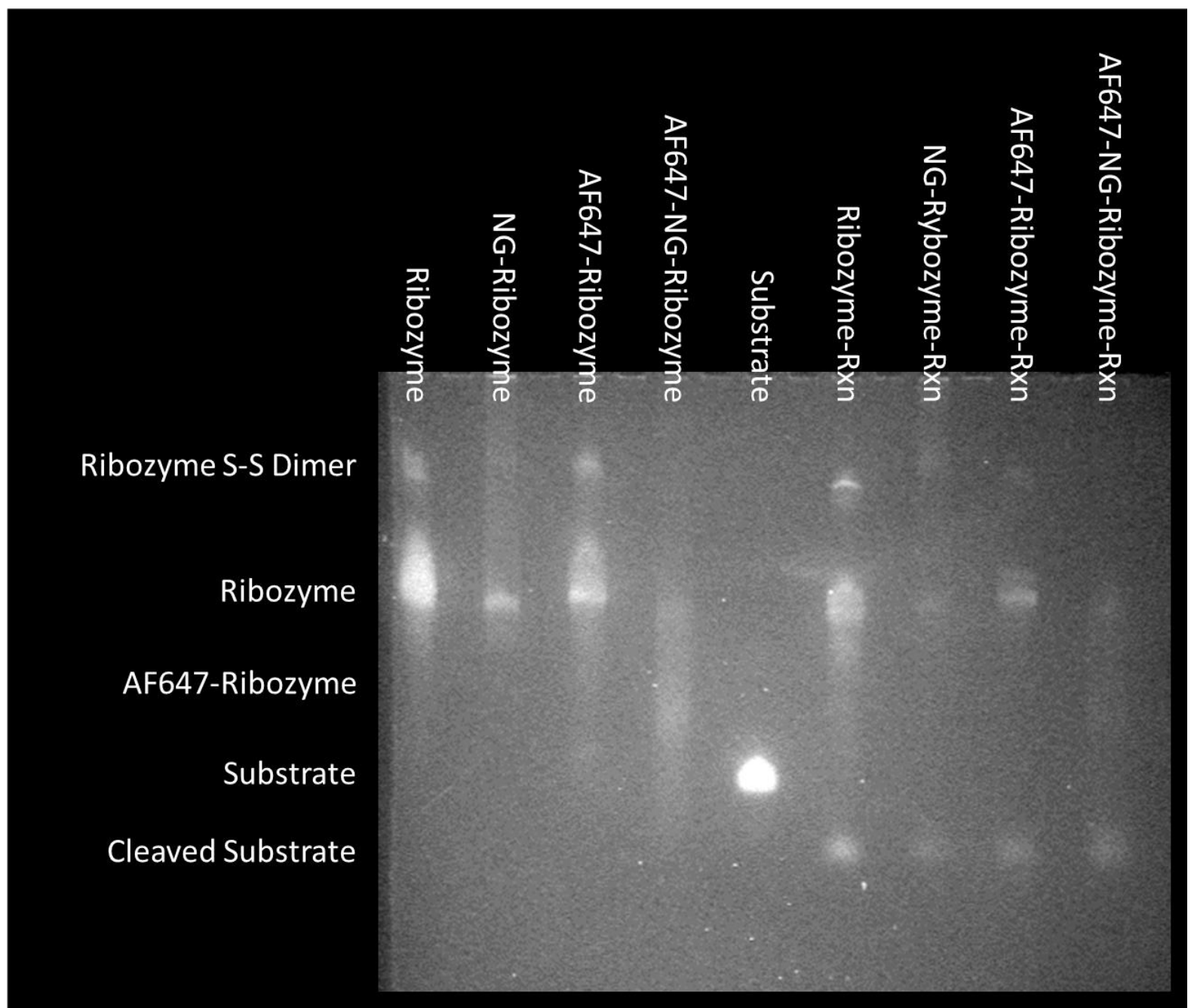

Figure SF4: UV illumination image of nondenaturing PAGE in $1 \times$ TBE running buffer at $10 \mathrm{~V} / \mathrm{cm}$ stained with Sybr Green following manufacturer's protocol showing assembly and catalytic competence of the Hammerhead RNA construct used in Scenario II. In all lanes, 'Ribozyme' consists of catalytic core annealed to Cy3-labeled substrate in $20 \mathrm{mM}$ PBS at a 1:1.1 ratio of Substrate:RNA catalytic core. Cleavage reactions were performed by incubating ribozyme in 100 $\mathrm{mM} \mathrm{Mg}^{2+}$ for 2 hours. Lane numbering from the left; Lane 1: unlabeled ribozyme. Lane 2: ribozyme bound to $2 \mathrm{~nm}$ Au NP. Lane 3: AF647 labeled ribozyme. Lane 4: fully labeled ribozyme. Lane 5: labeled substrate strand only. Lane 6: unlabeled ribozyme after incubation with $\mathrm{Mg}^{2+}$. Lane 7: ribozyme bound to $2 \mathrm{~nm}$ AuNP after incubation with $\mathrm{Mg}^{2+}$. Lane 8: AF647 labeled ribozyme after incubation with $\mathrm{Mg}^{2+}$. Lane 9: AF647 labeled ribozyme bound to $2 \mathrm{~nm}$ AuNP after incubation with $\mathrm{Mg}^{2+}$. NG = nano-gold particle. Image contrast enhanced in Microsoft PowerPoint. 


\section{Scattering Contribution to Lifetime Measurements}
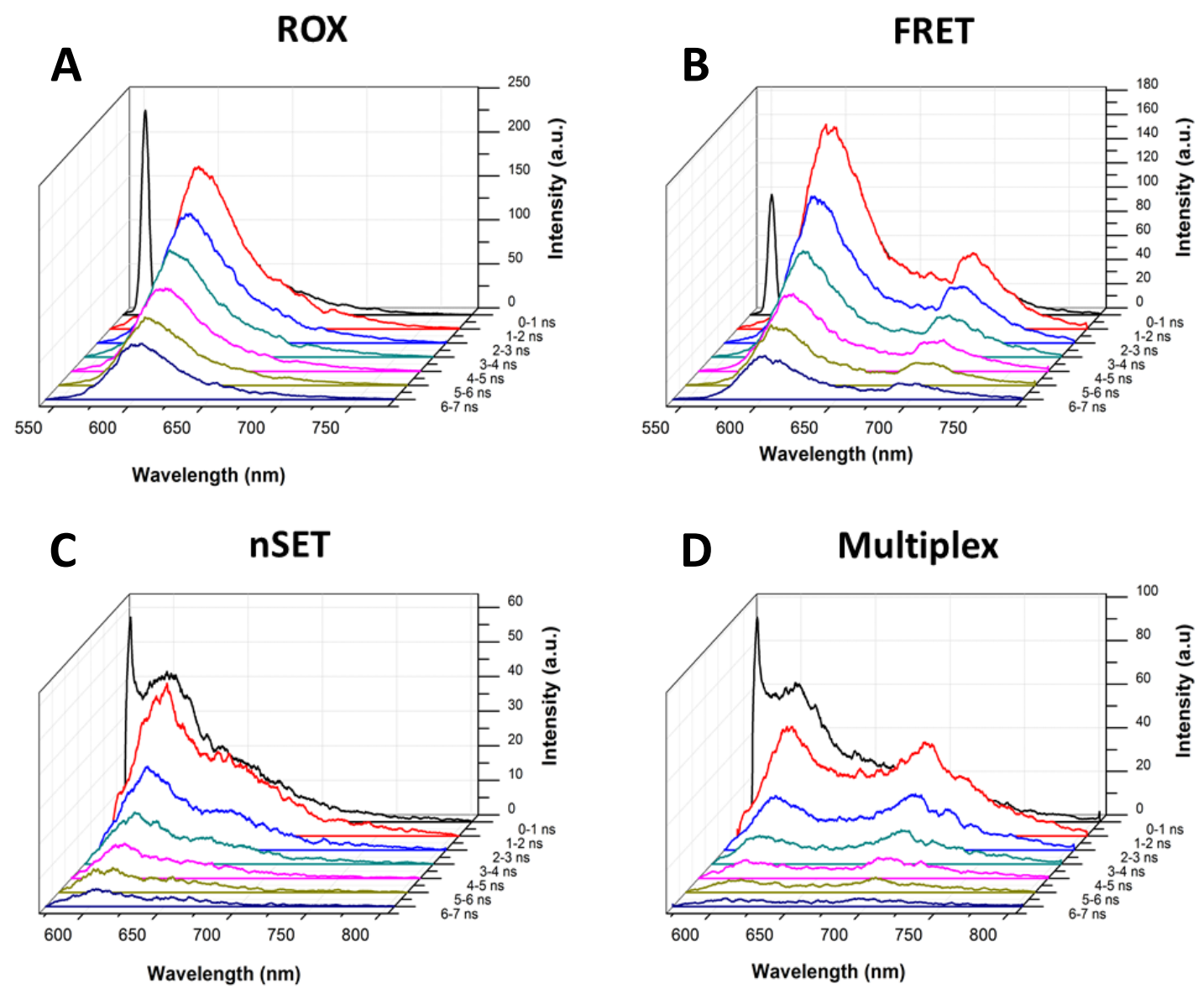

\section{E Scatter: 10nm Au}

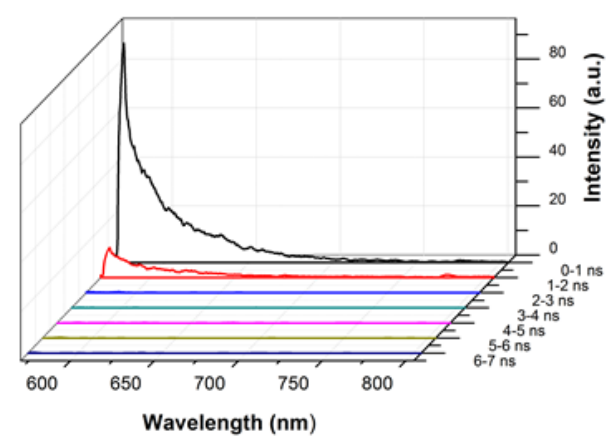

Figure SF5: 3D Streak-Camera decay profiles showing the time-dependent signal of the excitation pulse scattering from the $10 \mathrm{~nm}$ gold nanoparticles used in Scenario I. All spectra were collected in $20 \mathrm{mM}$ PBS (pH 6.5) and were excited at $560 \mathrm{~nm}$. A) Decay of free ROXlabeled DNA. The scattering of the pulse from the sample cuvette is visible as a distinct and well-separated peak at $560 \mathrm{~nm}$ that is below detection after $1 \mathrm{~ns}$. B) Decay of the dual labeled dsDNA FRET complex. As in panel A, the excitation pulse is a well resolved peak at $560 \mathrm{~nm}$ and below detection after $\sim 1$ ns. C) Decay of ROX-labeled DNA bound to 10nm AuNPs. Scattering of 
the excitation pulse from the gold particles can be seen as a much larger portion of the signal due to strong quenching of the ROX dye, and the pulse persists for $>1 \mathrm{~ns}$. D) Decay of duel labeled dsDNA FRET complex bound to $10 \mathrm{~nm}$ AuNP. As in C, scattering of the excitation pulse from the gold particles comprises a much larger portion of the signal due to strong quenching of the ROX dye, and the pulse can be seen to persist for longer than $1 \mathrm{~ns}$. E) Collected scattering signal from a sample containing free $10 \mathrm{~nm}$ AuNPs only. The broadened scattering signal can be clearly distinguished and the persistence of the signal out to $\sim 2$ ns can be seen.

\section{IIII. FRET-Only and SET-Only data}

\section{A. Absorbance and Emission Spectra}

Scenario I

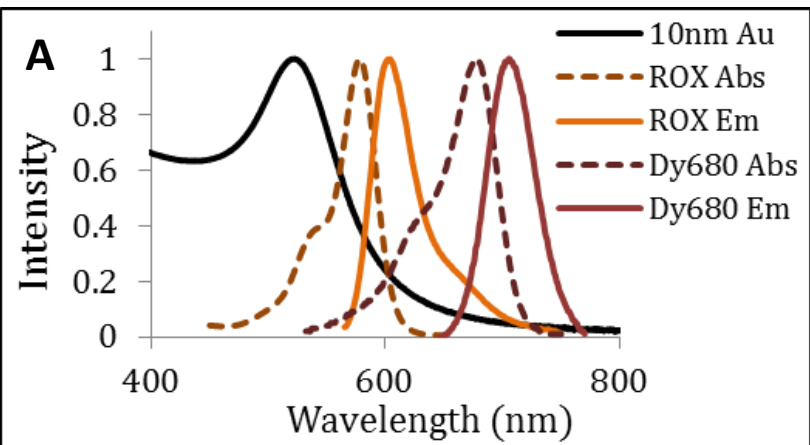

Scenario II

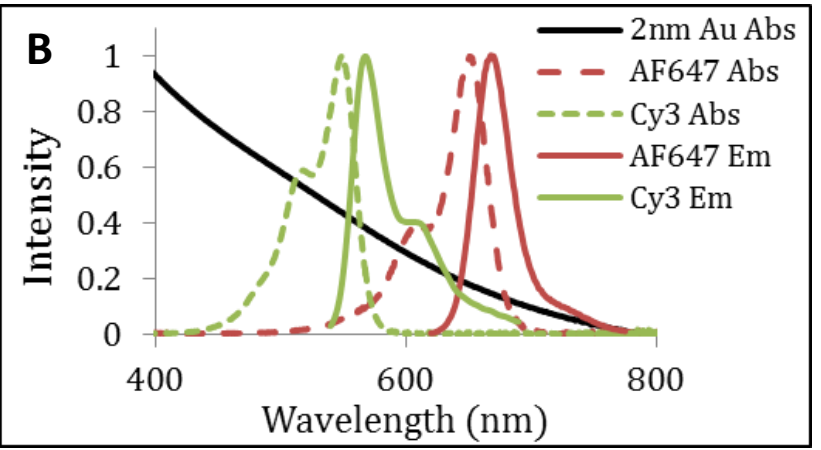

Figure SF6: A) Normalized absorbance of $10 \mathrm{~nm}$ AuNP overlapped with emission and absorbance profiles of ROX and DyLt680 dye labels. B) Normalized absorbance of $2 \mathrm{~nm}$ AuNP overlapped with emission and absorbance profiles of Cy3 and AF647 labels.

\section{B. Scenario I - DNA FRET and SET Decays}

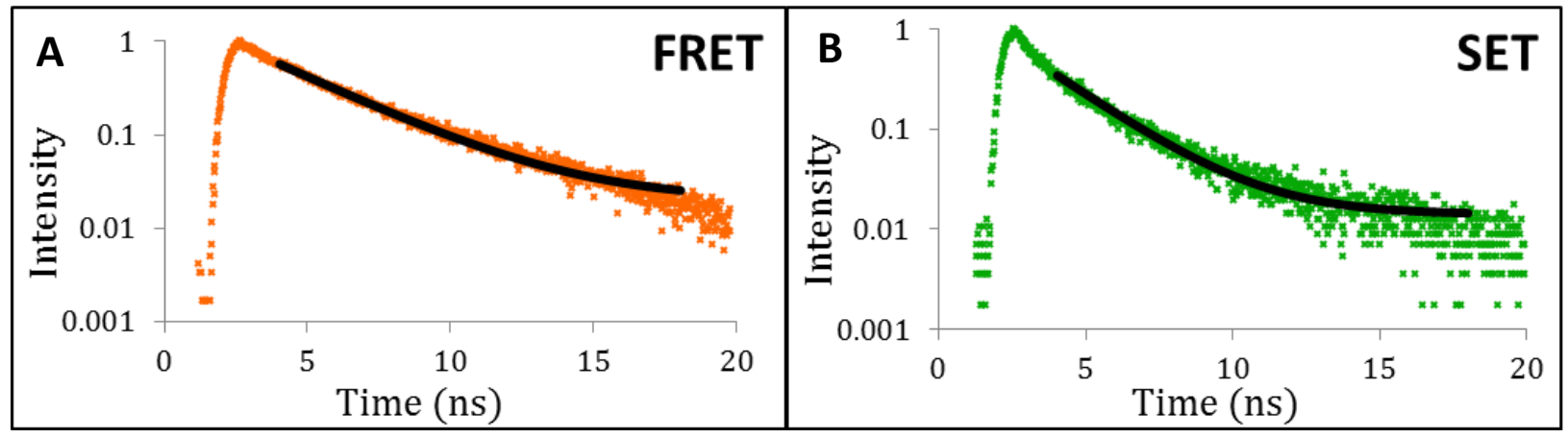

Figure SF7: A) Normalized fluorescence decay of ROX undergoing FRET on the dsDNA ROX-DyLt680 complex in the absence of $10 \mathrm{~nm}$ AuNP. B) Normalized fluorescence decay of ROX undergoing SET on a dsDNA strand labeled only with ROX and 10nm Au (no DyLt680). Excitation was at $560 \mathrm{~nm}$. 


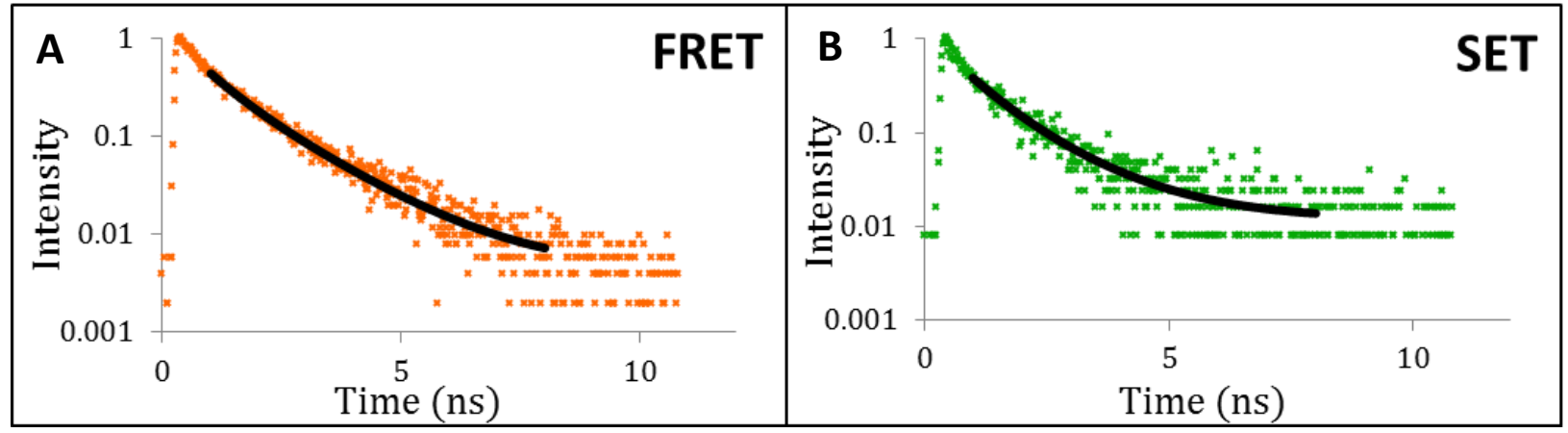

Figure SF8: A) Normalized fluorescence decay of Cy3 undergoing FRET on the AF647 labeled Hammerhead ribozyme complex annealed to a Cy3-labeled substrate in the absence of AuNP. B) Normalized fluorescence decay of Cy3 undergoing SET on a Hammerhead complex labeled only with Cy3-substrate and $2 \mathrm{~nm}$ Au labeled ribozyme (no AF647).

\section{Hammerhead RNA FRET and SET Results}

\begin{tabular}{|c|ccccccc}
\hline SET-only & $\left.\mathrm{d}_{0}(\AA)\right)$ & $\boldsymbol{\tau}^{\prime}(\mathrm{ns})$ & $\boldsymbol{\tau}_{\text {Th }}^{\prime}(\mathrm{ns})$ & Eff $_{\text {Exp }}\left(1-\frac{\tau^{\prime}}{\tau_{0}}\right)$ & Eff $_{\text {Th }}\left(\frac{R_{0}{ }^{n}{ }_{0}{ }^{n}+R^{n}}{n}\right)$ & $\mathrm{r}_{\text {Exp }}(\AA)$ & $\mathrm{r}_{\text {Th }}(\AA)$ \\
\hline ROX & 138 & 2.15 & 2.47 & 0.51 & 0.44 & 137 & 147 \\
DyLt680 & 71 & 1.68 & 1.68 & - & 0.00 & - & 218 \\
\hline Cy3 & 48 & 0.70 & 0.64 & 0.55 & 0.59 & 46 & 44 \\
AF647 & 45 & 0.37 & 0.38 & 0.73 & 0.74 & 35 & 35
\end{tabular}

Table ST1: Experimental and theoretical values for constructs undergoing SET only (no FRET). Dashes are used as entries when the corresponding data are not applicable. Here DyLt680 is not acting as a SET donor, and so does not experience any experimentally measured quenching value. It is only possible to set a lower bound for the distance between DyLt680 and AuNP, such that the absence of measureable energy transfer places the dye label at $>190 \AA$ from the AuNP surface.

\begin{tabular}{|c|ccccccc}
\hline FRET-only & $\left.\mathrm{R}_{0}(\AA)\right)$ & $\boldsymbol{\tau}^{\prime}(\mathrm{ns})$ & $\boldsymbol{\tau}_{\text {Th }}^{\prime}(\mathrm{ns})$ & Eff $_{\text {Exp }}\left(1-\frac{\tau^{\prime}}{\tau_{0}}\right)$ & $\mathbf{E f f}_{\text {Th }}\left(\frac{R_{0}{ }^{n}{ }_{R_{0}}{ }^{n}+R^{n}}{n}\right)$ & $\mathrm{r}_{\text {Exp }}(\AA)$ & $\mathrm{r}_{\text {Th }}(\AA)$ \\
\hline ROX & 67 & 3.05 & 2.74 & 0.30 & 0.37 & 77 & 73 \\
Cy3 & 51 & 0.61 & 0.49 & 0.55 & 0.59 & 46 & 47
\end{tabular}

Figure ST2: Experimental and theoretical values for constructs undergoing FRET only (no SET). 


\section{Energy Transfer Calculations}

\section{A. Derivation of McSET Formula}

We begin with the assumption that SET $\left(k_{S}\right)$ and FRET $\left(k_{F}\right)$ rates remain unperturbed when multiplexed as designed. The observed efficiency of quenching for a multiplexed SET-FRET systems $\left(E_{S F}\right)$ is defined as the ratio of combined energy transfer rates $\left(k_{S}+k_{F}\right)$ to the sum of all decay rates $\left(k_{S}+k_{F}+1 / \tau_{0}\right)$, where $\tau_{0}$ is the donor lifetime in the absence of FRET or SET. Therefore:

$$
\begin{aligned}
E_{S F}= & \frac{k_{S}+k_{F}}{k_{S}+k_{F}+1 / \tau_{0}}=\frac{1}{1+\left(\tau_{0}\left(k_{S}+k_{F}\right)\right)^{-1}} \\
& \Rightarrow\left(k_{S}+k_{F}\right)=\tau_{0}^{-1}\left(E_{S F}^{-1}-1\right)^{-1}
\end{aligned}
$$

Rearrangement yields the following relations:

$$
\begin{aligned}
& k_{S}=\tau_{0}^{-1}\left(E_{S F}^{-1}-1\right)^{-1}-k_{F} \\
& k_{F}=\tau_{0}^{-1}\left(E_{S F}^{-1}-1\right)^{-1}-k_{S}
\end{aligned}
$$

Then, remembering that

$$
E_{S}=\frac{1}{1+\left(d / d_{0}\right)^{4}}=\frac{k_{S}}{k_{S}+\tau_{0}^{-1}}
$$

And

$$
E_{F}=\frac{1}{1+\left(R / R_{0}\right)^{6}}=\frac{k_{F}}{k_{F}+\tau_{0}^{-1}}
$$

we rearrange to retrieve:

$$
\begin{aligned}
& k_{S}=\tau_{0}^{-1}\left(d / d_{0}\right)^{-4} \\
& k_{F}=\tau_{0}^{-1}\left(R / R_{0}\right)^{-6}
\end{aligned}
$$

Inserting the relations from SE5 into SE3 yields:

$$
\begin{aligned}
& \left(d / d_{0}\right)^{-4}=\left(E_{S F}^{-1}-1\right)^{-1}-\left(R / R_{0}\right)^{-6} \\
& \left(R / R_{0}\right)^{-6}=\left(E_{S F}^{-1}-1\right)^{-1}-\left(d / d_{0}\right)^{-4}
\end{aligned}
$$


Finally, recognizing that

$$
\begin{gathered}
E_{S F}^{-1}=\left(1-\tau_{S F} / \tau_{0}\right)^{-1} \\
=>\left(E_{S F}^{-1}-1\right)^{-1}=\tau_{0} / \tau_{S F}-1
\end{gathered}
$$

we can now rearrange again to produce the final expressions:

$$
d=d_{0}\left[\left(\frac{\tau_{0}}{\tau^{\prime}}-1\right)-\left(\frac{R}{R_{0}}\right)^{-6}\right]^{-1 / 4}
$$

for the SET contact distances. And

$$
R=R_{0}\left[\left(\frac{\tau_{0}}{\tau^{\prime}}-1\right)-\left(\frac{d}{d_{0}}\right)^{-4}\right]^{-1 / 6}
$$

for FRET contact distances.

\section{B. Calculation of $d_{0}$ and $R_{0}$.}

For Surface Energy Transfer, calculations of $\mathrm{d}_{0}$ values are obtained from previously reported approaches.

$$
d_{0}=\frac{\alpha \lambda}{n_{m}}(A \phi)^{1 / 4}\left(\frac{n_{r}}{2 n_{m}}\left(1+\frac{\varepsilon_{1}^{2}}{\left|\varepsilon_{2}\right|^{2}}\right)\right)^{1 / 4}
$$

The value $d_{0}$ is the distance at which the probability of energy transfer is $50 \%$, in analogy to $R_{0}$ in FRET. The donor specific terms are: $\lambda$, the emission wavelength maximum for the donor, and $\Phi$, the quantum yield of the donor. The acceptor specific terms include $A$, the absorptivity of a gold nanoparticle; $\mathrm{n}_{r}$, the refractive index of the metal; and $\varepsilon_{2}$, the complex dielectric function of the metal which comprises real and imaginary components $\left(\varepsilon_{\mathbf{2}}=\boldsymbol{\varepsilon}_{\mathbf{2}}{ }^{\prime}+\mathrm{i} \boldsymbol{\varepsilon}_{\mathbf{2}}{ }^{\prime \prime}\right)$. Above, $\boldsymbol{\varepsilon}_{\mathbf{1}}$ is the solvent dielectric and $n_{m}$ is the index of refraction for the solvent. The orientation of the donor to the metal plasmon vector, $\boldsymbol{\alpha}$, is taken as the averaged orientation vector resulting in $\boldsymbol{\alpha}=$ $\left((9 / 2)^{1 / 4}\right) / 4 \pi$.

The A-term in SE10 can be expressed in terms of the absorptivity within a single nanoparticle in analogy to molecular absorption, can be formulated as:

$$
A_{n p}=10^{3} \ln (10)\left[\frac{\epsilon_{\lambda}\left(2 r_{c m}\left(\frac{2 r_{c m}}{\delta_{s k i n}}\right)\right)}{N_{A} V_{c m^{3}}}\right]
$$


where $\epsilon_{\lambda}$ is the extinction coefficient of the NP at the maximum emission wavelength of the donor (size dependent $\epsilon_{\lambda}$ values are reported for spherical AuNPs ${ }^{33,34}$ ), $r_{c m}$ is the radius of the $\mathrm{NP}$ in $\mathrm{cm}, \mathrm{N}_{\mathrm{A}}$ is Avogadro's number, and $\boldsymbol{V}_{\mathbf{c m}^{3}}$ is the volume of the particle in $\mathrm{cm}^{3} .\left(2 \mathrm{r}_{\mathrm{cm}} / \delta_{\text {skin }}\right)$ is a correction term to account for the depth into the particle which the donor electric field can penetrate.

For FRET, calculations of $R_{0}$ are obtained using the well-known FRET equations.

$$
R_{0}=0.2108\left[\kappa^{2} n^{-4} \phi_{0}\langle J\rangle\right]^{1 / 6}
$$

Analogous to $d_{0}$ in SET, $R_{0}$ is the distance at which the probability of energy transfer is $50 \% . \mathrm{k}^{2}$ is the orientation factor typically taken to be $2 / 3, \mathrm{n}$ is the refractive index of the media, and $\phi$ is the quantum yield of the donor. The J-overlap integral, $\langle J\rangle$, is obtained using the following integration:

$$
\langle J\rangle=\int F_{D}(\lambda) \varepsilon_{A}(\lambda) \lambda^{4} d \lambda
$$

$F_{D}(\lambda)$ is the emission of the donor normalized to an area of $1 . \varepsilon_{A}(\lambda)$ is the molar absorptivity of the acceptor, and $\lambda$ is wavelength in units of $\mathrm{nm}$. The units of $\langle\mathrm{J}\rangle$ in this formulation are $\mathrm{cm}^{2} \mathrm{~nm}^{4} / \mathrm{mol}$.

\section{Constants for SET and FRET calculations}

\begin{tabular}{|c|cccccccc|}
\hline Scenario I & $\lambda_{\text {Max }}^{a b s}$ & $\epsilon_{\text {Max }}^{\text {dye }}$ & $\lambda_{\text {Max }}^{\text {em }}$ & $\phi_{0}$ & $\epsilon_{\text {Max }}^{10 n m A u}$ & $n_{\text {AuNP }}$ & $\delta_{\text {AuNP }}$ & $\varepsilon_{\text {Max }}^{10 n m A u}$ \\
\hline ROX & $575 \mathrm{~nm}$ & 82,000 & $609 \mathrm{~nm}$ & 0.7 & $2.06 \times 10^{7}$ & 0.25 & $28.4 \mathrm{~nm}$ & $-11.5+i 2.7$ \\
DyLt680 & $680 \mathrm{~nm}$ & 140,000 & $710 \mathrm{~nm}$ & 0.21 & $4.61 \times 10^{6}$ & 0.13 & $25.6 \mathrm{~nm}$ & $-19.3+i 3.7$ \\
\hline
\end{tabular}

Table ST3: Values used in calculating $\mathrm{d}_{0}$ and $\mathrm{R}_{0}$ values for ROX and DyLt680 in Scenario I.

\begin{tabular}{|c|cccccccc|}
\hline Scenario II & $\lambda_{\text {Max }}^{\text {abs }}$ & $\epsilon_{\text {Max }}^{\text {dye }}$ & $\lambda_{\text {Max }}^{\text {em }}$ & $\phi_{0}$ & $\epsilon_{\text {Max }}^{2 n m A u}$ & $n_{\text {AuNP }}$ & $\delta_{\text {AuNP }}$ & $\varepsilon_{\text {Max }}^{2 n m A u}$ \\
\hline Cy3 & $550 \mathrm{~nm}$ & 136,000 & $563 \mathrm{~nm}$ & 0.15 & $2.98 \times 10^{5}$ & 0.37 & $33.2 \mathrm{~nm}$ & $-5.5+i 7.2$ \\
AF647 & $653 \mathrm{~nm}$ & 250,000 & $667 \mathrm{~nm}$ & 0.33 & $1.39 \times 10^{5}$ & 0.13 & $29.4 \mathrm{~nm}$ & $-11.2+i 9.8$ \\
\hline
\end{tabular}

Table ST4: Values used in calculating $d_{0}$ and $R_{0}$ values for Cy3 and AF647 in Scenario II. 